\title{
一个具有扩散和比例依赖响应函数捕食模型 的定性分析
}

彭锐 (1) * 王明新 (2)

(1) 三峡大学理学院非线性复杂系统研究所, 宜昌 443002

(2) 东南大学数学系, 南京 210018

*E-mail: pengrui_seu@163.com

收稿日期: 2006-11-03; 接受日期: 2007-11-01

湖北省教育厅科研基金 (批准号: Q200713001) 和国家自然科学基金 (批准号: 10726016, 10771032) 资助项目

摘要本文考虑了一个具有扩散项和比例依赖响应函数的捕食模型. 该模型带有齐次 Neumann 边界条件. 本文主要关心该反应扩散系统解的大时间行为及其对应的平衡态问 题. 首先通过构造各种 Lyapunov 函数, 讨论唯一的正常数平衡解的全局稳定性. 然后, 对于 平衡态问题, 建立了正平衡解上下界的先验估计, 并且导出了当物种的扩散系数很大或者很 小时非常数正平衡解的一些不存在性结果.

关键词捕食模型 扩散 比例依赖 平衡态 全局稳定性 不存在性

$\operatorname{MSC}(2000)$ 主题分类 $35 \mathrm{~J} 55,37 \mathrm{~B} 25,92 \mathrm{D} 25$

\section{1 引言}

生物数学的许多工作致力于研究描述各种动力系统, 特别是带有各种边界条件的竞争和 捕食相互作用过程的反应扩散方程组. 在现存的文献中, 大多数应用数学家的注意力集中于 所考查模型物种的动力学行为以及对应的平衡态 (steady-state) 的性质, 他们的研究工作既 有数值模拟方面的, 也有纯粹数学理论方面的. 特别地, 最近许多作者考虑了下面描述食物和 猎物相互作用的反应扩散方程组:

$$
\begin{cases}\frac{\partial u}{\partial t}-d_{1} \Delta u=u(\lambda-\alpha u)-f(u) v, & (x, t) \in \Omega \times(0, \infty), \\ \frac{\partial v}{\partial t}-d_{2} \Delta v=v\left(\delta-\frac{v}{u}\right), & (x, t) \in \Omega \times(0, \infty), \\ \partial_{\nu} u=\partial_{\nu} v=0, & (x, t) \in \partial \Omega \times(0, \infty), \\ u(x, 0)=u_{0}(x)>0, \quad v(x, 0)=v_{0}(x) \geqslant 0, \not \equiv 0, & x \in \bar{\Omega},\end{cases}
$$


其中 $u(x, t)$ 和 $v(x, t)$ 分别表示食物和猎物的物种密度, $\Omega \subset \mathbb{R}^{n}$ 是一个具有光滑边界 $\partial \Omega$ 的 有界区域, $\nu$ 则是定义在边界 $\partial \Omega$ 上的向外的单位法向量, 且记 $\partial_{\nu}=\partial / \partial \nu$. 常数 $d_{i}(i=1,2)$ 是对应于食物 $u$ 和猎物 $v$ 的扩散系数 (也称为扩散率), $\lambda$ 和 $\delta$ 代表各自的内在净增长率, $\alpha$ 则表示环境对食物的承载能力 (carrying capacity) 系数. $C^{1}$ - 函数 $f(u)$ 被称为食物依赖型的 响应函数 (functional response), 它描述系统中猎物的捕食行为, 而 $v / u$ 表示环境对猎物的承 载能力函数, 显然这意味着环境对猎物的承载能力是依赖于食物的. 在 (1.1) 中所出现的参数 都假定为正常数. 相容的初值函数 $u_{0}(x)$ 和 $v_{0}(x)$ 是定义在 $\bar{\Omega}$ 上的连续函数. 齐次 Neumann 边值条件说明系统 (1.1) 是自我封闭的, 即在边界 $\partial \Omega$ 上食物和猎物的进出流量为零. 关于该 模型更多的生物学背景, 有兴趣的读者可进一步参见文献 [1-9] 等.

当响应函数 $f(u)=\beta u$ (其中 $\beta$ 为一正常数) 时, 在文献 $[2,3]$ 中, Du, Hsu 以及 Wang 研 究了模型 (1.1). 此时的响应函数 $f(u)=\beta u$ 通常称之为 Leslie-Gower 型的响应函数 ${ }^{[10]}$. 特别 地, 在空间环境是均匀的情形 (亦即 $\alpha>0$ 是常值函数), 只要对参数 $\alpha$ 和 $\beta$ 强加适当的条件, 通过构造一个技巧性的 Lyapunov 函数, 他们获得了该模型唯一的正常数平衡解的全局稳定 性. 而且, 该文作者主要关心在空间非均匀环境下系统 (1.1) 的平衡态问题. 他们因此而发现 了关于模式生成 (pattern formation) 的一些有趣的生物现象. 关于这些内容的具体细节, 请 参见文献 $[2,3]$. 最近, 本文作者在文献 [11] 中获得了当猎物的扩散系数很小时, 该模型正常 数解的唯一性和稳定性, 从而改进了文献 [2] 中的一个结果. 在另外一个正在准备的工作 ${ }^{[12]}$ 中, $\mathrm{Du}$ 和本文作者继续研究系统 (1.1). 此时的 $\beta$ 是一个给定的非负函数, 表示食物具有保护 层. 我们试图揭示当猎物的增长率很大时保护层对该模型平衡态解的渐近性态的影响.

如果 $f(u)$ 是 Holling-Tanner 型 (也就是 Holling-II 型) 的响应函数, 即 $f(u)=\beta u /(m+u)$, 这里 $m$ 是一正常数, 本文作者以及 Yang 在文献 $[6,13,14]$ 中讨论了系统 $(1.1)$, 主要感兴趣 于解的大时间性态. 大致说来, 我们分析了该模型唯一的正常数平衡解的全局稳定性, 建立了 当扩散系数以及交错扩散系数变化时非常数正平衡解的存在性和不存在性结果.

最近, 在文献 [15] 中, 当 $f(u)$ 满足一般性的假设条件时, 即: $f(0)=0$, 且存在某一正常 数 $M$, 使得 $0<f_{u}(u) \leqslant M$ 对所有的 $u>0$ 成立, Ko 和 Ryu 研究了系统 (1.1). 他们也分析 了该模型唯一的正常数平衡解的全局稳定性, 且导出了非常数正平衡解的存在性和不存在性 结果.

在上面谈到的模型中, 响应函数 $f(u)$ 只与变量 $u$ 有关, 这是食物依赖型的响应函数, 说 明猎物的捕食行为仅仅由食物决定. 然而, 最近来自生物控制的一些数值模拟表明: 经典的 食物依赖型的捕食模型会与实际观察到的现象, 例如由 Rosenzweig ${ }^{[16]}$ 提出的著名的 “丰富 化悖论” (paradox of enrichment) 相矛盾. 另一方面, 越来越多的证据表明, 在一些特定的生态 环境下, 特别是当猎物不得不主动的寻找、共享以及掠夺食物时, 更合理的捕食模型应当是 所谓的具有比例依赖型的模型, 即响应函数是比例依赖的. 相关阐述可进一步参见文献 [17]).

基于上述考虑, 本文关心的捕食模型中猎物的捕食行为不同于前面提到的模型. 也就是 说, 我们将要提出的模型的响应函数由比例依赖的函数来刻画, 因此该模型考虑到在猎物捕 食过程中食物起到的更合理的影响. 我们试图确定该捕食模型解的大时间动力学行为, 为此, 将讨论食物和猎物是否共存, 甚至是否最终会在空间上均匀分布. 另外, 从动力系统的数学理 论的角度来讲, 该模型解的大时间行为在很大程度上依赖于其平衡态解的性质, 因此, 我们也 将探讨该模型所对应的平衡态问题解的定性性质. 
更确切地讲, 我们考虑模型 (1.1) 的响应函数是比例依赖型的, 从而导致处理如下的捕食 模型 (为简单起见, 我们在模型 (1.1) 中取 $\delta=1$ ):

$$
\begin{cases}\frac{\partial u}{\partial t}-d_{1} \Delta u=u(\lambda-u)-\frac{\beta u v}{u+m v}, & (x, t) \in \Omega \times(0, \infty), \\ \frac{\partial v}{\partial t}-d_{2} \Delta v=v\left(1-\frac{v}{u}\right), & (x, t) \in \Omega \times(0, \infty), \\ \partial_{\nu} u=\partial_{\nu} v=0, & (x, t) \in \partial \Omega \times(0, \infty), \\ u(x, 0)=u_{0}(x)>0, \quad v(x, 0)=v_{0}(x) \geqslant 0, \not \equiv 0, & x \in \bar{\Omega},\end{cases}
$$

这里的 $\beta$ 和 $m$ 分别表示猎物的捕食率和饱和率 (saturation rate). 关于比例依赖响应函数更 详细的生物学解释, 有兴趣的读者可以参见文献 [17-20] 以及其中的文献.

显然, 系统 (1.2) 有唯一的正常数平衡解 $(u, v)=\left(u^{*}, v^{*}\right)$ 当且仅当 $\beta<\lambda(1+m)$, 而且 $\left(u^{*}, v^{*}\right)$ 可显式表达为

$$
\left(u^{*}, v^{*}\right)=\left(\lambda-\frac{\beta}{1+m}, \lambda-\frac{\beta}{1+m}\right)
$$

下文若没有特殊说明, 则总是假定 $\beta<\lambda(1+m)$ 成立, 从而保证了 $\left(u^{*}, v^{*}\right)$ 的存在性.

对于系统 (1.2), 我们将讨论解的逗留性质, 同时, 利用两种不同的 Lyapunov 函数证明 $\left(u^{*}, v^{*}\right)$ 的全局稳定性. 而且, 我们的理论分析表明, 为了获得如下 3 种不同的模型: 模型 (1.1) 中 $f(u)=\beta u$ 和 $f(u)=u /(m+u)$, 以及系统 (1.2) 正常数平衡解的全局稳定性, 需要选取 不同类型的 Lyapunov 函数. 从而说明在文献 [15] 中作者对具有一般响应函数 $f(u)$ 的模型 (1.1) 所运用的 Lyapunov 函数在某些情形下不是最优的.

如前所述, 系统 (1.2) 的非负解的关于时间渐近行为与其对应的平衡态解密切相关. 为此, 这促使我们分析系统 (1.2) 的平衡态问题, 它满足

$$
\left\{\begin{array}{llll}
-d_{1} \Delta u=u(\lambda-u)-\frac{\beta u v}{u+m v}, & x \in \Omega ; & \partial_{\nu} u=0, & x \in \partial \Omega, \\
-d_{2} \Delta v=v\left(1-\frac{v}{u}\right), & x \in \Omega ; & \partial_{\nu} v=0, & x \in \partial \Omega .
\end{array}\right.
$$

显然, 系统 (1.3) 的非负解才具有真正的生物学意义. 对于该模型, 本文将建立其正解的先验 估计. 在此基础上, 利用不同的数学分析技巧, 也将讨论当扩散系数 $d_{1}$ 或者 $d_{2}$ 很大或者很小 时非常数正解的不存在性.

最后, 需要指出的是, 可以利用本文使用的一些数学方法研究在文献 [15] 中提出的一般 模型, 从而获得关于唯一的常数正平衡解的全局稳定性以及非常数正平衡解不存在性的一些 改进性的结果.

\section{$2\left(u^{*}, v^{*}\right)$ 关于系统 $(1.2)$ 的全局稳定性}

本节主要讨论 $\left(u^{*}, v^{*}\right)$ 对于系统 $(1.2)$ 的全局稳定性. 令 $(u(x, t), v(x, t))$ 是系统 $(1.2)$ 唯 一的解. 显而易见, $(u(x, t), v(x, t))$ 总是整体存在且取正值, 即 $u(x, t), v(x, t)>0$ 对所有的 $x \in \bar{\Omega}$ 以及 $t>0$ 都成立. 


\section{1 系统 (1.2) 解的逗留性质}

本节讨论系统 $(1.2)$ 的解 $(u(x, t), v(x, t))$ 的逗留性质. 逗留性质说明, 不论两物种的扩散 率多大或者多小, 它们在任意时间和任意空间位置都是共存的.

命题 2.1 假定 $\beta=\lambda m$ 且 $m<1$ 或者 $\beta<\lambda m$ 成立, 则对于任意的 $0<\varepsilon \ll 1$, 存在足 够大的 $t_{0} \gg 1$, 使得当 $x \in \bar{\Omega}$ 且 $t \geqslant t_{0}$ 时, 系统 $(1.2)$ 的解 $(u(x, t), v(x, t))$ 满足

$$
K-\varepsilon<u(x, t), v(x, t)<\lambda+\varepsilon,
$$

其中, $K$ 由下面的表达式给定:

$$
K=\frac{1}{2}\left\{\lambda(1-m)+\sqrt{\lambda^{2}(1-m)^{2}+4 \lambda(\lambda m-\beta)}\right\} .
$$

证明 对任意给定的 $0<\varepsilon \ll 1$, 利用系统 $(1.2)$ 的第 1 个方程式以及拋物型方程的比 较原理得知, 存在较大的 $t_{0} \gg 1$, 使得 $u(x, t)<\lambda+\varepsilon$ 对所有的 $x \in \bar{\Omega}$ 和 $t \geqslant t_{0}$ 成立. 因此, $v(x, t)$ 是下面问题的一个下解:

$$
\left\{\begin{array}{l}
\frac{\partial z}{\partial t}-d_{2} \Delta z=\frac{\lambda+\varepsilon-z}{\lambda+\varepsilon} z, \quad(x, t) \in \Omega \times\left(t_{0}, \infty\right), \\
\partial_{\nu} z=0, \quad(x, t) \in \partial \Omega \times\left(t_{0}, \infty\right) ; \quad z\left(x, t_{0}\right)=v\left(x, t_{0}\right)>0, \quad x \in \bar{\Omega} .
\end{array}\right.
$$

我们也定义 $v(t)$ 是

$$
\left\{\begin{array}{l}
w_{t}=\frac{\lambda+\varepsilon-w}{\lambda+\varepsilon} w, \quad t \in\left(t_{0}, \infty\right), \\
w\left(t_{0}\right)=\max _{\bar{\Omega}} v\left(x, t_{0}\right)>0
\end{array}\right.
$$

的唯一的正解. 于是, $v(t)$ 为初边值问题 (2.1) 的上解, 从而比较原理导出

$$
v(x, t) \leqslant v(t), \quad \forall x \in \bar{\Omega}, t \geqslant t_{0} .
$$

由于 $\lim _{t \rightarrow \infty} v(t)=\lambda+\varepsilon$, 故存在 $t_{1}>t_{0}$ 满足

$$
v(x, t) \leqslant v(t)<\lambda+\varepsilon, \quad \forall x \in \bar{\Omega}, \quad t \geqslant t_{1} .
$$

因而, 再次借助于模型 (1.1) 的第 1 个方程式, $u(x, t)$ 是

$$
\begin{cases}\frac{\partial z}{\partial t}-d_{1} \Delta z=\frac{-z^{2}+[\lambda-m(\lambda+\varepsilon)] z+(\lambda m-\beta)(\lambda+\varepsilon)}{z+m(\lambda+\varepsilon)} z, & (x, t) \in \Omega \times\left(t_{1}, \infty\right), \\ \partial_{\nu} z=0, \quad(x, t) \in \partial \Omega \times\left(t_{1}, \infty\right) ; \quad z\left(x, t_{1}\right)=u\left(x, t_{1}\right)>0, \quad x \in \bar{\Omega}\end{cases}
$$

的一个上解. 又记 $u(t)$ 是问题

$$
\left\{\begin{array}{l}
w_{t}=\frac{-w^{2}+[\lambda-m(\lambda+\varepsilon)] w+(\lambda m-\beta)(\lambda+\varepsilon)}{w+m(\lambda+\varepsilon)} w, \quad t \in\left(t_{1}, \infty\right), \\
w\left(t_{1}\right)=\min _{\bar{\Omega}} u\left(x, t_{1}\right)>0
\end{array}\right.
$$

的解. 显然, $u(t)$ 是 $(2.2)$ 式的下解. 进而, 比较原理给出

$$
u(x, t) \geqslant u(t), \quad \forall x \in \bar{\Omega}, t \geqslant t_{1} .
$$

如果 $\beta=\lambda m$ 且 $m<1$ 或者 $\beta<\lambda m$ 成立, 简单的分析表明

$$
\lim _{t \rightarrow \infty} u(t)=\frac{1}{2}\left\{\lambda-m(\lambda+\varepsilon)+\sqrt{[\lambda-m(\lambda+\varepsilon)]^{2}+4(\lambda m-\beta)(\lambda+\varepsilon)}\right\} .
$$


因此, 对于任意给定的 $0<\varepsilon \ll 1$, 存在 $t_{2}>t_{1}$, 使得当 $\forall x \in \bar{\Omega}$ 以及 $t \geqslant t_{2}$ 时,

$$
u(x, t)>\frac{1}{2}\left\{\lambda(1-m)+\sqrt{\lambda^{2}(1-m)^{2}+4 \lambda(\lambda m-\beta)}\right\}-\varepsilon=K-\varepsilon .
$$

类似的, 利用上面的结果, 可以验证 $v(x, t)$ 满足命题 2.1 中的下界. 命题证毕.

\section{$2.2\left(u^{*}, v^{*}\right)$ 的全局稳定性}

本节试图导出 $\left(u^{*}, v^{*}\right)$ 关于系统 (1.2) 的一些全局稳定性结论. 从生物学意义上讲, 我们 的结果意味着, 在关于 $\lambda, \alpha, \beta$ 以及 $m$ 的某些参数范围, 不论食物和猎物的扩散系数怎样, 当 时间趋于无穷大时, 它们总是在空间上密度均匀分布.

定理 2.1 假定 $\beta=\lambda m<(1+m)^{2} K$ 且 $m<1$ 或者 $\beta<\min \left\{\lambda m,(1+m)^{2} K\right\}$ 成立. 另外, 若

$$
\left(\lambda+u^{*}\right) \beta<(1+m)^{2} K\left(K+u^{*}\right),
$$

这里, $K$ 由命题 2.1 中给定, 则 $\left(u^{*}, v^{*}\right)$ 关于系统 (1.2) 是全局稳定的. 特别地, 这说明此时系 统 (1.3) 不存在非常数正解.

证明 令 $(u(x, t), v(x, t))$ 是系统 (1.2) 的解. 为证明论断, 需要构造一个 Lyapunov 函数. 为此, 采用文献 [2] 中提出的 Lyapunov 函数

$$
\begin{aligned}
& W(u, v)=\int \frac{u^{2}-\left(u^{*}\right)^{2}}{u^{2}} d u+\alpha \int \frac{v-v^{*}}{v} d v, \\
& E(t)=\int_{\Omega} W(u(x, t), v(x, t)) d x,
\end{aligned}
$$

其中 $\alpha$ 为一待定的正常数.

直接的计算给出

$$
\begin{aligned}
\frac{d E(t)}{d t}= & \int_{\Omega}\left\{W_{u}(u(x, t), v(x, t)) u_{t}+W_{v}(u(x, t), v(x, t)) v_{t}\right\} d x \\
= & \int_{\Omega}\left\{\frac{u^{2}-\left(u^{*}\right)^{2}}{u^{2}} d_{1} \Delta u+\alpha \frac{v-v^{*}}{v} d_{2} \Delta v\right\} d x \\
& +\int_{\Omega}\left\{\frac{\left(u+u^{*}\right)\left(u-u^{*}\right)}{u}\left(\lambda-u-\frac{\beta v}{u+m v}\right)+\alpha\left(v-v^{*}\right)\left(1-\frac{v}{u}\right)\right\} d x \\
= & -\int_{\Omega}\left\{d_{1} \frac{2\left(u^{*}\right)^{2}}{u^{3}}|\nabla u|^{2}+d_{2} \alpha \frac{v^{*}}{v^{2}}|\nabla v|^{2}\right\} d x \\
& +\int_{\Omega}\left\{\frac{\left(u+u^{*}\right)\left(u-u^{*}\right)}{u}\left(u^{*}+\frac{\beta v^{*}}{u^{*}+m v^{*}}-u-\frac{\beta v}{u+m v}\right)+\alpha\left(v-v^{*}\right)\left(\frac{v^{*}}{u^{*}}-\frac{v}{u}\right)\right\} d x \\
= & -\int_{\Omega}\left\{d_{1} \frac{2\left(u^{*}\right)^{2}}{u^{3}}|\nabla u|^{2}+d_{2} \alpha \frac{v^{*}}{v^{2}}|\nabla v|^{2}\right\} d x \\
& +\int_{\Omega} \frac{1}{u}\left\{\left(u+u^{*}\right)\left[-1+\frac{\beta v^{*}}{\left(u^{*}+m v^{*}\right)(u+m v)}\right]\left(u-u^{*}\right)^{2}\right. \\
& \left.+\left[-\frac{\beta u^{*}\left(u+u^{*}\right)}{\left(u^{*}+m v^{*}\right)(u+m v)}+\alpha\right]\left(u-u^{*}\right)\left(v-v^{*}\right)-\alpha\left(v-v^{*}\right)^{2}\right\} d x \\
= & -\int_{\Omega}\left\{d_{1} \frac{2\left(u^{*}\right)^{2}}{u^{3}}|\nabla u|^{2}+d_{2} \alpha \frac{v^{*}}{v^{2}}|\nabla v|^{2}\right\} d x+\int_{\Omega} \frac{1}{u}\left\{\left(u+u^{*}\right)\left[-1+\frac{\beta}{(1+m)(u+m v)}\right] \xi^{2}\right. \\
& \left.+\left[-\frac{\beta\left(u+u^{*}\right)}{(1+m)(u+m v)}+\alpha\right] \xi \eta-\alpha \eta^{2}\right\} d x,
\end{aligned}
$$

这里, $\xi=u-u^{*}$ 和 $\eta=v-v^{*}$. 
如果能够找到正常数 $\alpha$ 以及 $t_{0}$, 使得对所有的 $x \in \bar{\Omega}$ 和 $t \geqslant t_{0}$, 系统 (1.2) 的任意解 $(u(x, t), v(x, t))$ 满足

$$
\left(u+u^{*}\right)\left[-1+\frac{\beta}{(1+m)(u+m v)}\right] \xi^{2}+\left[-\frac{\beta\left(u+u^{*}\right)}{(1+m)(u+m v)}+\alpha\right] \xi \eta-\alpha \eta^{2} \leqslant 0,
$$

则有当 $t \geqslant t_{0}$ 时 $E^{\prime}(t) \leqslant 0$, 从而断言 $E(t)$ 为系统 (1.2) 的 Lyapunov 函数.

为验证二次型 $(2.5)$ 对所有的 $\xi$ 和 $\eta$ 成立, 只要要求

$$
\left[\alpha-\frac{\beta\left(u+u^{*}\right)}{(1+m)(u+m v)}\right]^{2}+4\left(u+u^{*}\right)\left[-1+\frac{\beta}{(1+m)(u+m v)}\right] \alpha<0 .
$$

同时注意到, $(2.5)$ 式是负的, 除非 $\xi=\eta=0$.

进一步地, 容易检验 $(2.6)$ 式等价于下面的式子成立:

$$
\beta<(1+m)(u+m v)
$$

和

$$
\alpha+\beta(1+m)^{-1}\left(u+u^{*}\right)(u+m v)^{-1}<2\left(u+u^{*}\right)^{1 / 2} \alpha^{1 / 2} .
$$

假定 $\beta=\lambda m$ 且 $m<1$ 或者 $\beta<\lambda m$ 成立. 利用命题 2.1 , 显然 (2.7) 和 (2.8) 式成立, 如 果

$$
\beta<(1+m)^{2} K
$$

以及

$$
\alpha+\beta\left(\lambda+u^{*}\right)(1+m)^{-2} K^{-1}<2\left(K+u^{*}\right)^{1 / 2} \alpha^{1 / 2},
$$

因此, 假设条件 (2.3) 保证了存在需要的 $\alpha$, 使得 (2.10) 式成立.

从上面的分析得知, $E(t)$ 是系统 (1.2) 的一个 Lyapunov 函数. 结合命题 2.1, 标准的论 述说明, $(u(x, t), v(x, t)) \rightarrow\left(u^{*}, v^{*}\right)$ 在 $\left[L^{\infty}(\Omega)\right]^{2}$ 中成立, 从而在假设条件下, $\left(u^{*}, v^{*}\right)$ 关于系统 (1.2) 是全局稳定的. 至此, 定理证毕.

下面将借助于文献 [4] 提出的 Lyapunov 函数获得 $\left(u^{*}, v^{*}\right)$ 全局稳定的另一个结果.

定理 2.2 假定 $\beta=\lambda m<(1+m)^{2} K$ 且 $m<1$ 或者 $\beta<\min \left\{\lambda m,(1+m)^{2} K\right\}$ 满足, 另外, 若

$$
\beta<\frac{\lambda(1+m)}{2},
$$

则 $\left(u^{*}, v^{*}\right)$ 关于系统 $(1.2)$ 是全局稳定的.

证明 为在条件 (2.11) 下证明所需结果, 我们选取的 Lyapunov 函数如下:

$$
\begin{aligned}
& W(u, v)=\int \frac{u-u^{*}}{u^{2}} d u+\alpha \int \frac{v-v^{*}}{v} d v, \\
& E(t)=\int_{\Omega} W(u(x, t), v(x, t)) d x,
\end{aligned}
$$

这里的 $\alpha>0$ 待定.

正如定理 2.1 中的计算直接得到

$$
\begin{aligned}
\frac{d E(t)}{d t}= & -\int_{\Omega}\left\{d_{1} \frac{2 u^{*}-u}{u^{3}}|\nabla u|^{2}+d_{2} \alpha \frac{v^{*}}{v^{2}}|\nabla v|^{2}\right\} d x \\
& +\int_{\Omega} \frac{1}{u}\left\{\left[-1+\frac{\beta}{(1+m)(u+m v)}\right] \xi^{2}+\left[-\frac{\beta}{(1+m)(u+m v)}+\alpha\right] \xi \eta-\alpha \eta^{2}\right\} d x,
\end{aligned}
$$


其中, $\xi=u-u^{*}$ 和 $\eta=v-v^{*}$.

同前所述, 容易验证, 如果不等式 $(2.7), u<2 u^{*}$ 且

$$
\alpha+\beta(1+m)^{-1}(u+m v)^{-1}<2 \alpha^{1 / 2}
$$

满足, 则 $E^{\prime}(t) \leqslant 0$ 对所有的 $t \geqslant t_{0}$ 成立, 因而 $\left(u^{*}, v^{*}\right)$ 是全局稳定的.

利用命题 2.1 并结合不等式 $\beta<(1+m)^{2} K$, 显然对于某个正常数 $\alpha,(2.13)$ 式是成立的. 而且, 再次利用命题 2.1, (2.12) 式意味着对所有的 $x \in \bar{\Omega}$ 和 $t \geqslant t_{0}$ 有 $u<2 u^{*}$. 从而, 我们的 分析证实了在题设条件下, 定理 2.2 成立. 至此, 定理证毕.

注 2.1 我们观察到, 如果 $m<1$, 则有 $\lambda(1-m) \leqslant K<\lambda$; 若 $\beta=\lambda m$, 则有 $K=$ $\lambda(1-m)$. 因而, 当下面情形之一发生: (1) $\lambda$ 充分大;(2) $m$ 足够大; (3) $\beta$ 足够小;(4) $\beta=\lambda m$ 且 $m^{2}+m^{3}<1$, 则由定理 2.1 以及定理 2.2 知 $\left(u^{*}, v^{*}\right)$ 是系统 $(1.2)$ 的全局吸引子.

注 2.2 需要指出, 定理 2.1 中的条件 (2.3) 和定理 2.2 中的条件 (2.11) 是互不包含的. 事实上, 当 $m$ 充分大时, 满足条件 (2.3) 的参数范围比满足 (2.11) 式的大. 另一方面, 若选取 $m=1$ 然后令 $\lambda=\theta \beta$, 其中 $\theta>1$, 仔细的计算表明, 当 $\theta$ 大于但接近于 $(2+\sqrt{5}) / 4$ 时, 定理 2.1 和定理 2.2 中的共同假设满足, 但此时满足条件 (2.3) 的参数范围严格地被满足 (2.11) 式 的参数范围所包含.

注 2.3 如果选取下面众所周知的 Lyapunov 函数:

$$
W(u, v)=\int \frac{u-u^{*}}{u} d u+\alpha \int \frac{v-v^{*}}{v} d v,
$$

其中 $\alpha$ 为待定正常数, 则类似于定理 2.1 的分析, 可以断言: $\left(u^{*}, v^{*}\right)$ 是全局稳定的, 只要 定理 2.1 中的条件 $(2.3)$ 被

$$
\lambda \beta<(1+m)^{2} K^{2}
$$

所替代. 由于 $K<\lambda$, 容易观察到, 定理 2.1 中的条件 (2.3) 包含了条件 (2.15). 从而, 为获取 $\left(u^{*}, v^{*}\right)$ 的全局稳定性, 我们发现, Lyapunov 函数 (2.4) 优于 Lyapunov 函数 (2.14).

注 2.4 为证明系统 (1.1) 唯一的正常数平衡解的全局稳定性, 当 $f(u)=\beta u$ 时, 文献 [2] 证实了 Lyapunov 函数 (2.4) 要比 Lyapunov 函数 (2.12) 以及 (2.14) 有效得多; 另一方面, 在文 献 [13] 中我们指出, 若 $f(u)=\beta u /(m+u)$, 则 Lyapunov 函数 (2.12) 比其他的 Lyapunov 函数 更合适. 然而, 就带有比例依赖的响应函数的模型而言, 注 2.2 和 2.3 告诉我们, Lyapunov 函 数 (2.4) 和 (2.12) 能够导出使得 $\left(u^{*}, v^{*}\right)$ 全局稳定的不同条件. 这里的叙述也说明, 文献 [15] 中选取的 Lyapunov 函数在某些情形并不是最优的.

\section{3 系统 (1.3) 的正解的先验估计}

从现在起, 我们的目的是考察平衡态问题 (1.3). 本节将获得系统 (1.3) 正解正的上下界 的先验估计. 首先能断言下面的结果:

定理 3.1 假定 $\beta \leqslant \lambda m$, 则对于任意给定的 $\varepsilon>0$, 存在一仅依赖于 $\varepsilon, \lambda, \beta, m$ 以及 $\Omega$ 的 正常数 $\mathrm{C}$, 使得当 $d_{1}, d_{2} \geqslant \varepsilon$ 时, 系统 (1.3) 任意的正解 $(u, v)$ 满足 $\mathrm{C}<u, v<\lambda$.

证明 简单的比较论述表明: $u(x), v(x)<\lambda$. 因此, 只需要验证 $(u, v)$ 的下界即可. 我们 采用反证法. 假定定理 3.1 的结论不成立, 于是存在序列 $\left\{\left(d_{1, i}, d_{2, i}\right)\right\}_{i=1}^{\infty}$ 且 $d_{1, i}, d_{2, i} \geqslant \varepsilon$ 以及 对应于 $\left(d_{1}, d_{2}\right)=\left(d_{1, i}, d_{2, i}\right)$ 的满足系统 $(1.3)$ 的正解 $\left(u_{i}, v_{i}\right)$, 使得

$$
\min _{\bar{\Omega}} u_{i}(x) \rightarrow 0 \text { 或者 } \min _{\bar{\Omega}} v_{i}(x) \rightarrow 0 \text {, 当 } i \rightarrow \infty \text { 时, }
$$


同时 $\left(u_{i}, v_{i}\right)$ 满足

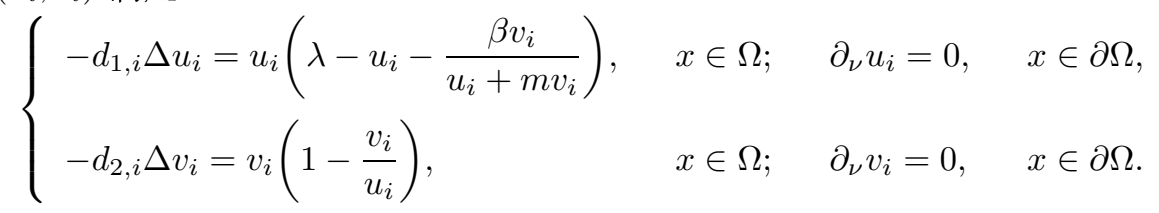

利用 Harnack 不等式 ${ }^{[21]}$ 知道, 存在不依赖于 $i$ 的正常数 $C$, 使得 $\max _{\bar{\Omega}} u_{i}(x) \leqslant C \min _{\bar{\Omega}} u_{i}(x)$. 另一方面, 借助于文献 [22] 中给出的最大值原理 (也可参见文献 [6] 中的引理 2.1) 可知, $\min _{\bar{\Omega}} u_{i}(x) \leqslant \min _{\bar{\Omega}} v_{i}(x)$, 从而必定有, 当 $i \rightarrow \infty$ 时 $\min _{\bar{\Omega}} u_{i}(x) \rightarrow 0$, 故 $u_{i}(x) \rightarrow 0$ 在 $\bar{\Omega}$ 上 一致成立. 注意到, 利用 $(3.2)$ 式的第 2 个方程式, 文献 [22] 的最大值原理导出 $\max _{\bar{\Omega}} v_{i}(x) \leqslant$ $\max _{\bar{\Omega}} u_{i}(x)$, 因而, 当 $i \rightarrow \infty$ 时 $v_{i}(x) \rightarrow 0$ 也在 $\bar{\Omega}$ 上一致成立.

首先考虑 $\left(d_{1, i}, d_{2, i}\right) \rightarrow\left(d_{1}, d_{2}\right)$ 且 $d_{1}, d_{2} \in[\varepsilon, \infty)$ 的情形. 定义

则 $\left(\hat{u}_{i}, w_{i}\right)$ 解

$$
\hat{u}_{i}=\frac{u_{i}}{\left\|u_{i}\right\|_{\infty}}, \quad w_{i}=\frac{v_{i}}{\left\|u_{i}\right\|_{\infty}}, \quad h_{i}=\frac{w_{i}}{\hat{u}_{i}+m w_{i}},
$$

$$
\left\{\begin{array}{llll}
-d_{1, i} \Delta \hat{u}_{i}=\hat{u}_{i}\left(\lambda-u_{i}-\beta h_{i}\right), & x \in \Omega ; & \partial_{\nu} \hat{u}_{i}=0, & x \in \partial \Omega, \\
-d_{2, i} \Delta w_{i}=w_{i}\left(1-\frac{w_{i}}{\hat{u}_{i}}\right), & x \in \Omega ; & \partial_{\nu} w_{i}=0, & x \in \partial \Omega .
\end{array}\right.
$$

由于 $h_{i}$ 是有界的, 从而存在 $h_{i}$ 的子列, 仍记为其自身, 使得在弱的意义下 $h_{i} \rightarrow h$ 在 $L^{2}(\Omega)$ 中成立且 $h \in L^{\infty}(\Omega)$. 因此, 利用标准的椭圆型方程的正则理论以及嵌入定理, 由 (3.3) 式的 第 1 个方程式, 若有必要再取其子列, 可以假定 $\hat{u}_{i} \rightarrow \hat{u}$ 在 $C^{1}(\bar{\Omega})$ 中成立, 同时 $\|\hat{u}\|_{\infty}=1$. 而 且, $\hat{u}$ 满足

$$
-d_{1} \Delta \hat{u}=\hat{u}(\lambda-\beta h), \quad x \in \Omega ; \quad \partial_{\nu} \hat{u}=0, \quad x \in \partial \Omega .
$$

由于 $\|\hat{u}\|_{\infty}=1$, Harnack 不等式意味着在 $\bar{\Omega}$ 上有 $\hat{u}>0$.

另一方面, 注意到对所有的 $i$ 有 $w_{i} \leqslant 1$, 利用标准的椭圆型方程正则性理论, 从 (3.3) 式 的第 2 个方程式, 可以假定 $w_{i} \rightarrow w$ 在 $C^{1}(\bar{\Omega})$ 中成立, 并且 $\|w\|_{\infty} \leqslant 1$, 以及 $w$ 是方程

$$
-d_{2} \Delta w=w\left(1-\frac{w}{\hat{u}}\right), x \in \Omega ; \quad \partial_{\nu} w=0, \quad x \in \partial \Omega
$$

的一个非负解. 假定 $w=0$. 在 $\Omega$ 上积分 (3.3) 式的第 2 个方程得到

$$
\int_{\Omega} w_{i}\left(1-\frac{w_{i}}{\hat{u}_{i}}\right) d x=0
$$

从而, 存在点列 $x_{i} \in \bar{\Omega}$, 使得 $w_{i}\left(x_{i}\right)=\hat{u}_{i}\left(x_{i}\right)$ 对每一个 $i \geqslant 1$ 都成立. 然而, 由于 $\hat{u}_{i} \rightarrow \hat{u}$ 在 $C^{1}(\bar{\Omega})$ 中成立且在 $\bar{\Omega}$ 上有 $\hat{u}>0$, 这与当 $i \rightarrow \infty$ 时 $w_{i} \rightarrow w=0$ 在 $C^{1}(\bar{\Omega})$ 成立相矛盾. 从而, 这意味着 $w$ 是 (3.5) 式的一个正解. 于是, 我们的分析显然说明当 $i \rightarrow \infty$ 时,

$$
h_{i} \rightarrow h=\frac{w}{\hat{u}+m w}
$$

在 $C^{1}(\bar{\Omega})$ 中成立. 结果, (3.4) 式等价于问题

$$
-d_{1} \Delta \hat{u}=\hat{u}\left(\lambda-\frac{\beta w}{\hat{u}+m w}\right), \quad x \in \Omega ; \quad \partial_{\nu} \hat{u}=0, \quad x \in \partial \Omega .
$$

在 $\Omega$ 上积分方程 $(3.6)$ 导出

$$
\int_{\Omega} \hat{u}\left(\lambda-\frac{w}{\hat{u}+m w}\right) d x=\int_{\Omega} \hat{u} \frac{\lambda \hat{u}+(\lambda m-\beta) w}{\hat{u}+m w} d x=0 .
$$


鉴于 $\beta \leqslant \lambda m$, 这也是不可能的.

若 $\left(d_{1, i}, d_{2, i}\right) \rightarrow\left(d_{1}, \infty\right)$ 且 $d_{1} \in[\varepsilon, \infty)$ 的情形发生, 记号 $\hat{u}_{i}$ 和 $w_{i}$ 如前. 类似的论述给 出 $\left(\hat{u}_{i}, w_{i}\right) \rightarrow(\hat{u}, w)$ 在 $C^{1}(\bar{\Omega}) \times C^{1}(\bar{\Omega})$ 中成立, 而且 $w=c$ 是一正常数. 另外, 方程 $(3.6)$ 仍 然满足, 故由 (3.7) 式导出矛盾. 若 $\left(d_{1, i}, d_{2, i}\right) \rightarrow\left(\infty, d_{2}\right)$ 且 $d_{2} \in[\varepsilon, \infty]$. 在此情形, 容易证明 $\left(\hat{u}_{i}, w_{i}\right) \rightarrow(1,1)$ 在 $C^{1}(\bar{\Omega}) \times C^{1}(\bar{\Omega})$ 中成立, 且 $(3.7)$ 式有效. 矛盾再次发生. 因此, 整个定理证 毕.

下面将给出当扩散系数 $d_{2}$ 很小时系统 (1.3) 正解的先验估计. 首先叙述一个预备性的结 果, 该结果可能是平凡的, 但为了方便读者参考, 在此仍给出详细证明.

引理 3.2 假定 $\mu>0$ 是一常数, $a(x)$ 是定义在 $\bar{\Omega}$ 上的一连续的正函数, 则问题

$$
-\Delta z=\mu z\left(1-a^{-1}(x) z\right), \quad x \in \Omega ; \quad \partial_{\nu} z=0, \quad x \in \partial \Omega
$$

有唯一的正解 $z_{\mu}$, 且当 $\mu \rightarrow \infty$ 时 $z_{\mu} \rightarrow a(x)$ 在 $\bar{\Omega}$ 上一致地成立.

证明 问题 (3.8) 正解的存在性和唯一性是众所周知的. 下面仅给出当 $\mu \rightarrow \infty$ 时 $z_{\mu}$ 的 渐近行为的分析.

由于 $C^{2}(\bar{\Omega})$ 在 $C(\bar{\Omega})$ 中是稠密的, 可以假定 $a(x) \in C^{2}(\bar{\Omega})$. 记 $d(x)=d(x, \partial \Omega)$ 且对于某正 常数 $c$ 有 $|\nabla a|<c$. 注意到, $\partial \Omega$ 是光滑的, 从而 $d(x)$ 是 $C^{2}$ 的且当 $x \in \partial \Omega$ 时满足 $\partial_{\nu} d(x)<0$.

现在, 我们试图构造问题 (3.8) 的一对上下解. 为此, 对于任意给定的 $\varepsilon>0$, 令

$$
\bar{z}=(1+\varepsilon) a(x)+f(x), \quad \underline{z}=(1-\varepsilon) a(x)-f(x),
$$

其中 $f(x)$ 满足:

(i) $f(x) \in C^{2}(\bar{\Omega})$, 且在 $\bar{\Omega}$ 上有 $0 \leqslant f(x) \leqslant \varepsilon$;

(ii) 当 $x$ 接近 $\partial \Omega$ 时有 $f(x)=\frac{\varepsilon}{2}-M d(x)$;

(iii) 对所有的 $x \in \partial \Omega$ 有 $-(1 \pm \varepsilon) c-M \partial_{\nu} d(x) \geqslant 0$, 其中 $M$ 是一给定的较大常数.

在上面的要求下, 对于充分大的 $\mu$, 简单的计算表明

$$
-\Delta \bar{z} \geqslant \mu \bar{z}\left(1-a^{-1}(x) \bar{z}\right), \quad x \in \Omega ; \quad \partial_{\nu} \bar{z} \geqslant 0, \quad x \in \partial \Omega,
$$

以及

$$
-\Delta \underline{z} \leqslant \mu \underline{z}\left(1-a^{-1}(x) \underline{z}\right), \quad x \in \Omega ; \quad \partial_{\nu} \underline{z} \leqslant 0, \quad x \in \partial \Omega .
$$

这些不等式说明, 只要 $\mu$ 足够大, $\bar{z}$ 和 $\underline{z}$ 分别为问题 (3.8) 的上下解. 因此, 问题 (3.8) 正解的 唯一性保证 $\underline{z} \leqslant z_{\mu} \leqslant \bar{z}$, 从而, 对任意小的 $\varepsilon$, 若 $\mu$ 充分大, 则有

$$
(1-\varepsilon) a(x)-\varepsilon \leqslant z_{\mu} \leqslant(1+\varepsilon) a(x)+\varepsilon .
$$

这意味着当 $\mu \rightarrow \infty$ 时, $z_{\mu} \rightarrow a(x)$ 在 $\bar{\Omega}$ 上一致成立. 引理证毕.

接下来, 对于当 $\lambda \neq \beta /(1+m)$ 时更一般的情形, 我们建立当 $d_{2}$ 很小时系统 (1.3) 正解 的先验估计.

定理 3.2 假定 $\lambda, \beta, m$ 固定且 $\lambda \neq \beta /(1+m)$, 则对于任意给定的 $\varepsilon_{0}>0$, 存在仅依赖 于 $\varepsilon_{0}, \lambda, \beta, m$ 以及 $\Omega$ 的正常数 $\varepsilon$ 及 $\underline{\mathrm{C}}$, 使得当 $\varepsilon_{0} \leqslant d_{1}$ 和 $0<d_{2} \leqslant \varepsilon$ 时, 系统 (1.3) 任意的正 解 $(u, v)$ 满足 $\mathrm{C}<u, v<\lambda$.

证明 根据定理 3.1 , 只要找到 $(u, v)$ 的下界即可. 再次使用反证法. 假定定理 3.2 的结 论错误, 则存在 $\varepsilon_{0}>0$ 以及序列 $\left\{\left(d_{1, i}, d_{2, i}\right)\right\}_{i=1}^{\infty}$ 且满足 $\varepsilon_{0} \leqslant d_{1, i}$ 和 $0<d_{2, i} \rightarrow 0$, 以及对应 于 $\left(d_{1}, d_{2}\right)=\left(d_{1, i}, d_{2, i}\right)$ 的满足系统 $(1.3)$ 的正解 $\left(u_{i}, v_{i}\right)$, 使得 $(3.1)$ 和 $(3.2)$ 式成立. 
正如定理 3.1 的证明, 当 $i \rightarrow \infty$ 时必定有 $u_{i}(x)$ 以及 $v_{i}(x)$ 在 $\bar{\Omega}$ 上一致地趋近于零. 记 $\hat{u}_{i}$ 和 $w_{i}$ 以及 $h_{i}$ 如前. 若 $d_{1, i} \rightarrow d_{1} \in\left[\varepsilon_{0}, \infty\right)$, 则根据引理 3.1 , 类似于定理 3.1 的论述, 若有 必要取其子列, 有 $\left(\hat{u}_{i}, w_{i}\right) \rightarrow(\hat{u}, \hat{u})$ 在 $\bar{\Omega}$ 上一致地成立, 而且 $\hat{u}$ 是问题

$$
-d_{1} \Delta \hat{u}=\hat{u}\left(\lambda-\frac{\beta}{1+m}\right), \quad x \in \Omega ; \quad \partial_{\nu} \hat{u}=0, \quad x \in \partial \Omega
$$

的正解. 这表明 $\lambda=\beta /(1+m)$, 矛盾. 若 $d_{1, i} \rightarrow \infty$ 情形发生, 容易证明, $\left(\hat{u}_{i}, w_{i}\right) \rightarrow(\hat{u}, w)=$ $(1,1)$ 在 $\bar{\Omega}$ 上一致成立, 且 $(\hat{u}, w)$ 满足 $(3.7)$ 式, 这再次导出矛盾. 于是, 定理证毕.

\section{4 非常数正解的不存在性}

建立在第 3 节对系统 (1.3) 正解先验估计的基础上, 本节给出当扩散系数 $d_{1}$ 或者 $d_{2}$ 很 大或者 $d_{2}$ 很小时, 系统 (1.3) 非常数正解的不存在性结果. 为此, 我们将运用一些不同的数 学技巧.

\section{1 当 $d_{1}$ 很大时非常数正解的不存在性}

记 $\mu_{1}$ 是算子 $-\Delta$ 定义在 $\Omega$ 内且带有齐次 Neumann 边界条件的最小的正的特征值. 首 先, 利用能量积分方法导出如下的不存在性结果:

定理 4.1 假定 $\beta \leqslant \lambda m$ 且让 $\varepsilon_{1}$ 是一任意给定的正常数, 则存在正常数 $D_{1}=D_{1}(\lambda, \beta, m$, $\left.\varepsilon_{1}, \Omega\right)>0$, 使得当 $D_{1}<d_{1}$ 以及 $\varepsilon_{1}<d_{2}$ 时, 系统 (1.3) 不存在非常数正解.

证明 记 $(u, v)$ 是系统 (1.3) 的正解, 且 $\bar{g}=\frac{1}{|\Omega|} \int_{\Omega} g d x$. 于是, 对系统 (1.3) 的第 1 和第 2 个方程分别乘以 $(u-\bar{u}) / u$ 和 $(v-\bar{v}) / v$, 然后在 $\Omega$ 上积分并相加, 得到

$$
\begin{aligned}
\int_{\Omega}\{ & \left.\frac{d_{1} \bar{u}|\nabla u|^{2}}{u^{2}}+\frac{d_{2} \bar{v}|\nabla v|^{2}}{v^{2}}\right\} d x \\
= & \int_{\Omega}\left\{-(u-\bar{u})\left(u+\frac{\beta v}{u+m v}\right)-(v-\bar{v}) \frac{v}{u}\right\} d x \\
= & \int_{\Omega}\left\{-(u-\bar{u})\left(u+\frac{\beta v}{u+m v}-\bar{u}-\frac{\beta \bar{v}}{\bar{u}+m \bar{v}}\right)-(v-\bar{v})\left(\frac{v}{u}-\frac{\bar{v}}{\bar{u}}\right)\right\} d x \\
= & \int_{\Omega}\left\{\left[-1+\frac{\beta \bar{v}}{(u+m v)(\bar{u}+m \bar{v})}\right](u-\bar{u})^{2}+\left[\frac{-\beta \bar{u}}{(u+m v)(\bar{u}+m \bar{v})}+\frac{\bar{v}}{\bar{u} u}\right]\right. \\
\quad & \left.\quad(u-\bar{u})(v-\bar{v})-\frac{1}{u}(v-\bar{v})^{2}\right\} d x
\end{aligned}
$$

若 $\beta \leqslant \lambda m$ 且 $\varepsilon_{1}<d_{2}$, 则利用定理 3.1 和 Young 不等式可知

$$
\begin{aligned}
\int_{\Omega}\left\{d_{1}|\nabla u|^{2}+d_{2}|\nabla v|^{2}\right\} d x \leqslant C \int_{\Omega}\{ & (u-\bar{u})^{2}\left[-1+\frac{\beta \bar{v}}{(u+m v)(\bar{u}+m \bar{v})}+C(\varepsilon)\right] \\
& \left.+(v-\bar{v})^{2}\left[-\frac{1}{u}+\varepsilon\right]\right\} d x,
\end{aligned}
$$

对任意的 $\varepsilon>0$ 成立. 这里, $C$ 仅依赖于 $\lambda, \beta, m, \varepsilon_{1}, \Omega$, 而 $C(\varepsilon)$ 仅依赖于 $\lambda, \beta, m, \varepsilon_{1}, \Omega$ 以及 $\varepsilon$. 另一方面, 根据定理 3.1 , 可以选择 $0<\varepsilon \ll 1$ 仅依赖于 $\lambda, \beta, m, \varepsilon_{1}, \Omega$, 使得 $-1 / u+\varepsilon<0$. 因 此, 结合 (4.10) 式, 有

$$
\int_{\Omega}\left\{d_{1}|\nabla(u-\bar{u})|^{2}+d_{2}|\nabla(v-\bar{v})|^{2}\right\} d x \leqslant C(\varepsilon) \int_{\Omega}(u-\bar{u})^{2} d x .
$$

鉴于著名的 Poincaré 不等式

$$
\mu_{1} \int_{\Omega}(g-\bar{g})^{2} d x \leqslant \int_{\Omega}|\nabla(g-\bar{g})|^{2} d x
$$


由 (4.11) 式, 得到

$$
\mu_{1} \int_{\Omega}\left\{d_{1}(u-\bar{u})^{2}+d_{2}(v-\bar{v})^{2}\right\} d x \leqslant C(\varepsilon) \int_{\Omega}(u-\bar{u})^{2} d x .
$$

显然, 存在仅依赖于 $\lambda, \beta, m, \varepsilon_{1}, \Omega$ 的 $D_{1}$, 使得当 $d_{1}>D_{1}$ 时有 $(u, v)=(\bar{u}, \bar{v})=\left(u^{*}, v^{*}\right)$, 于是 定理 4.1 成立. 定理证毕.

借助于定理 3.2, 容易看出, 由定理 4.1 的证明也能够得到下面的结果:

定理 4.2 假定 $\lambda(1+m)>\beta$, 则存在仅依赖于 $\lambda, \beta, m$ 以及 $\Omega$ 的正常数 $\varepsilon_{2}$ 和 $D_{1}$, 使得 当 $D_{1}<d_{1}$ 和 $0<d_{2} \leqslant \varepsilon_{2}$ 时, 系统(1.3) 不存在非常数正解.

\section{2 当 $d_{2}$ 很大时非常数正解的不存在性}

下面建立当 $d_{2}$ 很大时, 系统 (1.3) 非常数正解的不存在性结果. 首先, 能量估计允许我们 得到

定理 4.3 假定 $\beta \leqslant \lambda m$ 且令 $\varepsilon_{3}$ 为一任意正数, 则存在 $D_{2}=D_{2}\left(\lambda, \beta, m, \varepsilon_{3}, \Omega\right)>0$, 使 得当 $\lambda+\varepsilon_{3}<\mu_{1} d_{1}$ 和 $D_{2}<d_{2}$ 时系统(1.3) 不存在非常数正解.

证明 假定 $(u, v)$ 是系统 (1.3) 的正解且记 $\bar{g}=\frac{1}{|\Omega|} \int_{\Omega} g d x$. 我们限制 $d_{1} \geqslant 1$. 对系统 (1.3) 的第 1 个方程乘以 $(u-\bar{u})$, 然后在 $\Omega$ 上积分并利用定理 3.1, 得到

$$
\begin{aligned}
d_{1} \int_{\Omega}|\nabla(u-\bar{u})|^{2} d x=\int_{\Omega}\{ & {\left[\lambda-(u+\bar{u})-\frac{\beta m v \bar{v}}{(u+m v)(\bar{u}+m \bar{v})}\right](u-\bar{u})^{2} } \\
& \left.-\frac{m u \bar{u}}{(u+m v)(\bar{u}+m \bar{v})}(u-\bar{u})(v-\bar{v})\right\} d x \\
\leqslant & (\lambda+\varepsilon) \int_{\Omega}(u-\bar{u})^{2} d x+C(\varepsilon) \int_{\Omega}(v-\bar{v})^{2} d x
\end{aligned}
$$

对仅依赖于 $\lambda, \beta, m$ 以及 $\Omega$ 的 $\varepsilon>0$ 成立.

类似地, 借助于系统 (1.3) 的第 2 个方程, 不难得到

$$
d_{2} \int_{\Omega}|\nabla(v-\bar{v})|^{2} d x \leqslant \varepsilon \int_{\Omega}(u-\bar{u})^{2} d x+C(\varepsilon) \int_{\Omega}(v-\bar{v})^{2} d x .
$$

因此, 相加 (4.12) 和 (4.13) 式, 利用 Poincaré不等式, 有

$$
\mu_{1} \int_{\Omega}\left\{d_{1}(u-\bar{u})^{2}+d_{2}(v-\bar{v})^{2}\right\} d x \leqslant(\lambda+\varepsilon) \int_{\Omega}(u-\bar{u})^{2} d x+C(\varepsilon) \int_{\Omega}(v-\bar{v})^{2} d x .
$$

若 $\mu_{1} d_{1}>\lambda+\varepsilon_{3}$, 则由 (4.14) 式容易看出, 存在 $D_{2}=D_{2}\left(\lambda, \beta, m, \varepsilon_{3}, \Omega\right)>0$, 使得当 $d_{2}>D_{2}$ 时, 系统 $(1.3)$ 仅有常数正解 $(u, v)=\left(u^{*}, v^{*}\right)$. 于是, 定理证毕.

下面, 在一定条件下, 利用隐函数定理改进定理 4.3. 为此, 首先需要给出当 $d_{2} \rightarrow \infty$ 时, 系统 (1.3) 正解的渐近性态.

引理 4.1 固定 $d_{1}, \lambda, \beta, m$, 假定 $\beta=\lambda m$ 且 $m<1$ 或者 $\beta<\lambda m$ 成立. 记 $\left(u_{i}, v_{i}\right)$ 是系统 (1.3) 对应于 $d_{2}=d_{2, i}$ 的正解, 且当 $i \rightarrow \infty$ 时有 $d_{2, i} \rightarrow \infty$, 则 $\left(u_{i}, v_{i}\right) \rightarrow\left(u^{*}, v^{*}\right)$ 在 $\left[C^{2}(\bar{\Omega})\right]^{2}$ 中成立.

证明 利用定理 3.1 和标准的嵌入定理以及椭圆型方程理论知, 存在 $\left(u_{i}, v_{i}\right)$ 的子列, 仍 记为自身, 使得当 $i \rightarrow \infty$ 时 $\left(u_{i}, v_{i}\right) \rightarrow(u, v)$ 在 $\left[C^{2}(\bar{\Omega})\right]^{2}$ 上成立. 而且, $v \equiv c$, 这里的 $c$ 是一 正常数且 $c \leqslant \lambda$, 且在 $\bar{\Omega}$ 上有 $0<u$. 同时 $(u, c)$ 解

$$
\left\{\begin{array}{l}
-d_{1} \Delta u=\frac{u}{u+m c}\left[-u^{2}+(\lambda-m c) u+c(\lambda m-\beta)\right], \quad x \in \Omega ; \quad \partial_{\nu} u=0, \quad x \in \partial \Omega, \\
\int_{\Omega}\left(1-\frac{c}{u}\right) d x=0 .
\end{array}\right.
$$


因此, 借助于文献 [6] 中的引理 3.2, (4.15) 式的第 1 个方程表明 $u$ 必定为一正常数, 从而利用 第 2 个方程有 $(u, v)=\left(u^{*}, v^{*}\right)$. 引理证毕.

利用上面的引理, 我们可以改进定理 4.3 .

定理 4.4 假定 $\beta=\lambda m$ 且 $m<1$ 或者 $\beta<\lambda m$, 令 $\varepsilon_{4}$ 是一任意正常数, 则存在一较大 的正常数 $D_{2}=D_{2}\left(\varepsilon_{4}, \lambda, \beta, m, \Omega\right)$, 使得当 $\varepsilon_{4}<d_{1}$ 以及 $D_{2}<d_{2}$ 时, 系统 (1.3) 不存在非常数 正解.

证明 根据定理 4.3, 对固定的且仅依赖于 $\lambda, \beta, m, \Omega$ 的较大常数 $D_{1}$, 存在 $D_{2}=D_{2}(\lambda, \beta$, $m, \Omega)$, 使得当 $d_{1}>D_{1}, d_{2}>D_{2}$ 时, 系统 (1.3) 没有非常数正解. 因此, 在下面的讨论中, 只 需要考虑 $d_{1} \in\left[\varepsilon_{4}, D_{1}\right]$ 的情形.

作如下的分解:

$$
v=w+\xi, \text { 其中 } \int_{\Omega} w d x=0 \text { 和 } \xi \in \mathbb{R}^{+} .
$$

观察到, 寻找系统 (1.3) 的正解等价于解下面的问题:

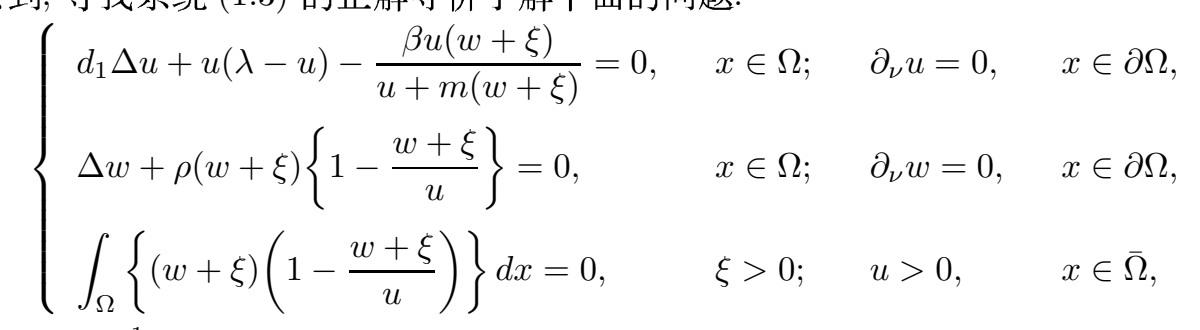

其中, $\rho=d_{2}^{-1}$. 显然, 对于任意的 $\rho>0,(u, w, \xi)=\left(u^{*}, 0, v^{*}\right)$ 都是问题 (4.16) 的解.

由上面的分析知, 为了验证我们的断言, 由有限覆盖论述可知, 只要证明对任意固定的 $\tilde{d}_{1} \in\left[\varepsilon_{4}, D_{1}\right]$, 存在较小的正常数 $\delta_{0}$, 使得当 $\rho \in\left(0, \delta_{0}\right), d_{1} \in\left(\tilde{d}_{1}-\delta_{0}, \tilde{d}_{1}+\delta_{0}\right)$ 时, $\left(u^{*}, 0, v^{*}\right)$ 是问题 (4.16) 唯一的正解.

为此, 定义

$$
W_{\nu}^{2,2}(\Omega)=\left\{g \in W^{2,2}(\Omega) \mid \partial_{\nu} g=0, \quad x \in \partial \Omega\right\}, \quad L_{0}^{2}(\Omega)=\left\{g \in L^{2}(\Omega) \mid \int_{\Omega} g d x=0\right\},
$$

且

则有

$$
\begin{aligned}
& F\left(d_{1}, \rho, u, w, \xi\right)=\left(f_{1}, f_{2}, f_{3}\right)(\rho, u, w, \xi) \\
& f_{1}\left(d_{1}, \rho, u, w, \xi\right)=d_{1} \Delta u+u(\lambda-u)-\frac{\beta u(w+\xi)}{u+m(w+\xi)}, \\
& f_{2}\left(d_{1}, \rho, u, w, \xi\right)=\Delta w+\rho(w+\xi)\left\{1-\frac{w+\xi}{u}\right\} \\
& f_{3}\left(d_{1}, \rho, u, w, \xi\right)=\int_{\Omega}\left\{(w+\xi)\left(1-\frac{w+\xi}{u}\right)\right\} d x
\end{aligned}
$$

$$
F: \mathbb{R}^{+} \times \mathbb{R}^{+} \times W_{\nu}^{2,2} \times\left(L_{0}^{2}(\Omega) \cap W_{\nu}^{2,2}(\Omega)\right) \times \mathbb{R}^{+} \rightarrow L^{2}(\Omega) \times L_{0}^{2}(\Omega) \times \mathbb{R},
$$

以及问题 (4.16) 等价于 $F\left(d_{1}, \rho, u, w, \xi\right)=0$. 而且, 当 $\rho=0$ 时, 问题 (4.16) 有唯一解 $(u, w, \xi)=\left(u^{*}, 0, v^{*}\right)$.

简单的计算表明,

$$
\Phi \equiv D_{(u, w, \xi)} F\left(\tilde{d}_{1}, 0, u^{*}, 0, v^{*}\right): W_{\nu}^{2,2} \times\left(L_{0}^{2}(\Omega) \cap W_{\nu}^{2,2}(\Omega)\right) \times \mathbb{R}^{+} \rightarrow L^{2}(\Omega) \times L_{0}^{2}(\Omega) \times \mathbb{R},
$$


这里

$$
\Phi(y, z, \tau)=\left(\begin{array}{c}
\tilde{d}_{1} \Delta y+\frac{2 \beta+\beta m-\lambda(1+m)^{2}}{(1+m)^{2}} y-\frac{\beta}{(1+m)^{2}}(z+\tau) \\
\Delta z \\
\int_{\Omega}\{y-(z+\tau)\} d x
\end{array}\right) .
$$

为利用隐函数定理, 需要验证映射 $\Phi$ 既单又满. 事实上, 假定 $\Phi(y, z, \tau)=(0,0,0)$, 则 $z \equiv$ 0 . 于是, 由关于 $y$ 的第 1 个方程知 $\tau \in \mathbb{R}$ 意味着 $y$ 必为一常数. 进而, 利用 $\Phi(y, z, \tau)=(0,0,0)$ 中的积分方程导出 $y=\tau$. 另一方面, 由于 $\beta \leqslant \lambda m$, 注意到 $2 \beta+\beta m-\lambda(1+m)^{2}<0$. 再次利 用关于 $y$ 的第 1 个方程, 容易验证 $y=\tau=0$. 从而, $\Phi$ 可逆. 类似地, 也可以证明 $\Phi$ 是满射.

根据隐函数定理知, 存在正常数 $\rho_{0}$ 以及 $\delta_{0}$, 使得对每个 $\rho \in\left[0, \rho_{0}\right]$ 和 $d_{1} \in\left(\tilde{d}_{1}-\delta_{0}, \tilde{d}_{1}+\delta_{0}\right)$, $\left(u^{*}, 0, v^{*}\right)$ 是 $F\left(d_{1}, \rho, u, w, \xi\right)=0$ 在 $B_{\delta_{0}}\left(u^{*}, 0, v^{*}\right)$ 中的唯一的解, 这里 $B_{\delta_{0}}\left(u^{*}, 0, v^{*}\right)$ 是 $W_{\nu}^{2,2}(\Omega) \times\left(L_{0}^{2}(\Omega) \cap W_{\nu}^{2,2}(\Omega)\right) \times \mathbb{R}$ 中的以 $\left(u^{*}, 0, v^{*}\right)$ 为球心 $\delta_{0}$ 为半径的球. 若有必要选取更 小的 $\rho_{0}$ 和 $\delta_{0}$, 借助于引理 4.1, 可以得到定理 4.4. 定理证毕.

\section{3 当 $d_{2}$ 很小时正解的不存在性}

本小节的目的是讨论当 $d_{2}$ 很小且 $\lambda(1+m)<\beta$ 时问题 (1.3) 不存在正解. 具体一些讲, 有

定理 4.5 让 $\lambda, \beta, m$ 固定且 $\lambda(1+m)<\beta$, 则对任意小的 $\varepsilon_{0}>0$, 存在仅依赖于 $\varepsilon_{0}, \lambda, \beta, m$ 以及 $\Omega$ 的正常数 $\varepsilon_{5}$, 使得当 $\varepsilon_{0} \leqslant d_{1}$ 以及 $0<d_{2} \leqslant \varepsilon_{5}$ 时, 问题 (1.3) 不存在 正解.

证明 假定我们的结论不对, 则存在 $\varepsilon_{0}>0$ 以及一列 $\left\{\left(d_{1, i}, d_{2, i}\right)\right\}_{i=1}^{\infty}$, 且满足 $\varepsilon_{0} \leqslant d_{1, i}$ 以及 $d_{2, i} \rightarrow 0$, 使得问题 $(1.3)$ 有正解 $\left(u_{i}, v_{i}\right)$ 对应于 $\left(d_{1}, d_{2}\right)=\left(d_{1, i}, d_{2, i}\right)$.

我们仅分析 $d_{1, i} \rightarrow d_{1} \in\left[\varepsilon_{0}, \infty\right)$ 的情形, 当 $d_{1, i} \rightarrow \infty$ 时也可类似验证. 利用引理 3.1 和 定理 3.2 , 正如定理 3.2 的论述, 可以假定 $\left(u_{i}, v_{i}\right) \rightarrow(u, u)$ 在 $\bar{\Omega}$ 中一致地成立, 而且 $u$ 是

$$
-d_{1} \Delta u=u(\lambda-\beta /(1+m)-u), \quad x \in \Omega ; \quad \partial_{\nu} u=0, x \in \partial \Omega
$$

的正解. 显然, 当 $\lambda(1+m)<\beta$ 时上面的方程没有正解. 从而定理成立. 证毕.

注 4.1 需要指出的是, 证明问题 (1.3) 的非常数正解的存在性似乎是比较困难的. 原因 在于我们不能够在弱的条件下得到问题 (1.3) 正解下界的先验估计, 从而不能利用诸如文献 $[6,18,19]$ 中的拓扑度理论来建立非常数正解的存在性.

致谢作者对两位审稿人对本文初稿的仔细阅读和有益的评论表示真诚的感谢.

\section{参考文献}

1 Braza P A. The bifurcation structure of the Holling-Tanner model for predator-prey interactions using two-timing. SIAM J Appl Math, 63: 889-904 (2003)

2 Du Y H, Hsu S B. A diffusive predator-prey model in heterogeneous environment. J Differential Equations, 203: 331-364 (2004)

3 Du Y H, Wang M X. Asymptotic behavior of positive steady-states to a predator-prey model. Proc Roy Soc Edinburgh Sect A, 136: 759-778 (2006) 
4 Hsu S B, Huang T W. Global stability for a class of predator-prey systems. SIAM J Appl Math, 55: 763-783 (1995)

5 May R M. Stability and Complexity in Model Ecosystems. Princeton, NJ: Princeton University Press, 1973

6 Peng R, Wang M X. Positive steady-states of the Holling-Tanner prey-predator model with diffusion. Proc Roy Soc Edinburgh Sect A, 135: 149-164 (2005)

7 Saez E, Gonzalez-Olivares E. Dynamics of a predator-prey model. SIAM J Appl Math, 59: 1867-1878 (1999)

8 Tanner J T. The stability and the intrinsic growth rates of prey and predator populations. Ecology, 56: 855-867 (1975)

9 Wollkind D J, Collings J B, Logan J A. Metastability in a temperature-dependent model system for predator-prey mite outbreak interactions on fruit flies. Bull Math Biol, 50: 379-409 (1988)

10 Holling C S, The functional response of invertebrate predators to prey density. Mem Ent Soc Can, 45: 3-60 (1965)

11 Peng R, Wang M X. Uniqueness and stability of steady states for a predator-prey model in heterogeneous environment. Proc Amer Math Soc, 136: 859-865 (2008)

12 Du Y H, Peng R, Wang M X. Effect of a certain protection zone in a diffusive prey-predator model. Preparation

13 Peng R, Wang M X. Global stability of the equilibrium of a diffusive Holling-Tanner prey-predator model. Appl Math Lett, 20: 664-670 (2007)

14 Peng R, Wang M X, Yang G Y. Stationary patterns of the Holling-Tanner prey-predator model with diffusion and cross-diffusion. Appl Math Comput, to appear

15 Ko W, Ryu K. Non-constant positive steady-states of a predator-prey system in homogeneous environment. J Math Anal Appl, 327: 539-549 (2007)

16 Rosenzweig M L. Paradox of enrichment: destabilization of exploitation systems in ecological time. Science, 171: 385-387 (1969)

17 Abrams P, Ginzburg L. The nature of predation: prey dependent, ratio-dependent, or neither. Trends Ecol Evol, 15: 337-341 (2000)

18 Pang P Y H, Wang M X. Qualitative analysis of a ratio-dependent predator-prey system with diffusion. Proc Roy Soc Edinburgh Sect A, 133: 919-942 (2003)

19 Pang P Y H, Wang M X. Strategy and stationary pattern in a three-species predator-prey model. $J$ Differential Equations, 200: 245-273 (2004)

20 Peng R, Shi J P, Wang M X. Stationary pattern of a ratio-dependent food chain model with diffusion. SIAM J Appl Math, 67: 1479-1503 (2007)

21 Lin C S, Ni W M, Takagi I. Large amplitude stationary solutions to a chemotais systems. J Differential Equations, 72: 1-27 (1988)

22 Lou Y, Ni W M. Diffusion, self-diffusion and cross-diffusion. J Differential Equations, 131: 79-131 (1996) 